\title{
An Improved Arcflow Model for the Skiving Stock Problem
}

John Martinovic, Maxence Delorme, Manuel Iori, and Guntram Scheithauer

\begin{abstract}
Because of the sharp development of (commercial) MILP software and hardware components, pseudo-polynomial formulations have been established as a viable tool for solving cutting and packing problems in recent years. Constituting a natural (but independent) counterpart of the well-known cutting stock problem, the one-dimensional skiving stock problem (SSP) asks for the maximal number of large objects (specified by some threshold length) that can be obtained by recomposing a given inventory of smaller items. In this paper, we introduce a new arcflow formulation for the SSP applying the idea of reflected arcs. In particular, this new model is shown to possess significantly fewer variables as well as a better numerical performance compared to the standard arcflow formulation.
\end{abstract}

Key words: Cutting and Packing, Skiving Stock Problem, Arcflow Model, ILP

\section{Introduction}

We consider a threshold length $L \in \mathbb{N}$, and $m \in \mathbb{N}$ item types that are specified by their length $l_{i}$ and frequency (of occurence) $b_{i}(i \in I:=\{1, \ldots, m\}$ ). Then, the onedimensional skiving stock problem (SSP) requires to recompose the given items in order to obtain a maximal number of objects each having a length at least $L$. Origi-

John Martinovic $(\bowtie) \cdot$ Guntram Scheithauer

Institut für Numerische Mathematik, Technische Universität Dresden, Germany

e-mail: \{john.martinovic, guntram.scheithauer\}@tu-dresden.de

Maxence Delorme

School of Mathematics, The University of Edinburgh, United Kingdom

e-mail:maxence.delormeded.ac.uk

Manuel Iori

DISMI, Università di Modena e Reggio Emilia, Italy

e-mail: manuel.iori@unimore.it 
nating from applications in paper recycling [7], such objectives are of high interest in industrial production [2, 14] and wireless communications [8] as well. The problem under consideration was introduced in [1] for the special case of highly heterogeneous input lengths and very small quantities $b_{i}(i \in I)$, respectively, and originally termed as the dual bin packing problem. Its current name goes back to Zak [14], who presented a first pattern-based modelling approach for arbitrary frequencies. Although this model is conjectured to possess a very tight LP relaxation, similiar to the cutting context [6, 10, 12, 13], the number of variables is exponential with respect to the number of items. Consequently, pseudo-polynomial models for the SSP have been established in literature [9] and were shown to exhibit an equally good relaxation. For a long time, research on these alternative formulations for discrete optimization problems was rather theoretically motivated; but especially after the publication of the famous book [11] by Nemhauser and Wolsey, also computational aspects (e.g., the strength of the considered models) became a central concern in IP modeling. In recent years, the sharp development of (commercial) MILP software and the rapid progress in terms of powerful hardware components have successively increased the scientific importance of pseudo-polynomial formulations for the solution of cutting and packing problems.

In this article, we introduce a new graph-theoretical ILP formulation for the SSP that is based on the idea of reflected arcs originally presented in [5]. The main novelty of this approach is to only consider half of the bin capacity (i.e., a significantly reduced set of vertices), so that any pattern is decomposed into two subpaths being connected by a reflected arc. Thereby, the number of arcs of the arcflow graph can be decreased considerably compared to the original formulation [9].

In the next section, we briefly review the standard arcflow approach for the SSP. Afterwards, the improved formulation and some related theoretical properties are presented. Then, the computational performance of both models is compared based on randomly generated instances. Finally, we summarize the main ideas of this paper and give an outlook on future research.

\section{The Arcflow Model}

Let $E=(m, l, L, b)$ with $l=\left(l_{1}, \ldots, l_{m}\right)^{\top} \in \mathbb{Z}_{+}$and $b=\left(b_{1}, \ldots, b_{m}\right)^{\top} \in \mathbb{Z}_{+}$denote an instance of the SSP. Without loss of generality, we may assume that $L>l_{1}>l_{2}>\ldots>l_{m} \geq 1$ is satisfied. Any combination of items is represented by a vector $a=\left(a_{1}, \ldots, a_{m}\right)^{\top} \in \mathbb{Z}_{+}^{m}$ (with $a_{i} \in \mathbb{Z}_{+}$counting the items of type $i \in I$ ), and is referred to as a pattern whenever $l^{\top} a \geq L$ holds. Note that considering the set $P^{\star}(E)$ of minimal patterns (where each appearing item is indeed necessary to ensure $\left.l^{\top} a \geq L\right)$ is sufficient. Let $\bar{v}:=\max \left\{l^{\top} a \mid a \in P^{\star}(E)\right\}$ describe the maximal length of a minimal pattern. Then, the graph $\mathscr{G}=(\mathscr{V}, \mathscr{E})$ with $\mathscr{E}=\left\{(p, q) \in \mathscr{V} \times \mathscr{V} \mid p<L, q-p \in\left\{l_{1}, \ldots, l_{m}\right\}\right\}$ and $\mathscr{V}=\{0,1, \ldots, \bar{v}\}$ can be used to model the SSP. Here, an arc $(p, q) \in \mathscr{E}$ indicates the positioning of an 
item of length $q-p=l_{j} \in\left\{l_{1}, \ldots, l_{m}\right\}$ at vertex $p \in \mathscr{V}$. For this approach, different improvements have been discussed in the literature - most importantly the theory of normal patterns or raster points [3, 4] (restricting $\mathscr{V}$ to only feasible combinations of the item lengths), and symmetry reductions by considering monotonically decreasing paths [9] - leading to a simpler graph $\mathscr{G}^{\prime}=\left(\mathscr{V}^{\prime}, \mathscr{E}^{\prime}\right)$, in general. Let $x_{p q}$ denote the flow along the $\operatorname{arc}(p, q) \in \mathscr{E}^{\prime}$; then the arcflow model [9] is given by:

\section{Arcflow model of the SSP}

$$
\begin{array}{ll} 
& z^{A F}=\max \sum_{(0, q) \in A^{+}(0)} x_{0 q} \\
& \sum_{(p, q) \in A^{-}(q)} x_{p q}=\sum_{(q, r) \in A^{+}(q)} x_{q r}, \quad q \in \mathscr{V}^{\prime}, 0<q<L, \\
& \sum_{(p, q) \in E(i)} x_{p q} \leq b_{i}, \\
x_{p q} \in \mathbb{Z}_{+}, & i \in I,
\end{array}
$$

Note that $A^{-}(q)$ and $A^{+}(q)$ model the incoming and emanating arcs of vertex $q \in$ $\mathscr{V}^{\prime}$, respectively, whereas $E(i)$ collects all arcs referring to an item of length $l_{i}$. Constraints (1) can be interpreted as a flow conservation, whereas conditions (2) ensure that the given item limitations are respected. Thus, the objective function maximizes the total flow within $\mathscr{G}^{\prime}$, that is, the total number of objects obtained.

\section{The Reflect Arcflow Model}

As explained in [5], the key idea of the improved approach is to start from the arcflow model, but to represent a bin by using half of its capacity twice. More specifically, each arc whose head would lie in the second half is reflected into the first half. Since skiving stock patterns can exhibit any length $L_{t} \in \mathscr{L}:=\left\{l^{\top} a \mid a \in P^{\star}(E)\right\}$ (which are identical to the sinks of $\mathscr{G}^{\prime}$ ), we usually would have to cope with multiple reflection points $L_{t} / 2$. Moreover, the elements of $\mathscr{L}$ are not known in advance and, hence, cannot be used to efficiently implement a reflect graph. Due to these difficulties, we introduce additional loss arcs in order to force any path (or pattern) to have exactly the length $L$. Then, [5, Algorithm 7] (with some very minor adaptations to the new context) can be applied to build the reflect graph $\mathscr{H}:=(\mathscr{U}, \mathscr{A})$ (for the SSP) with only one reflection point $r:=L / 2$. Indeed, we have that any arc $(u, v) \in \mathscr{E}^{\prime}$ (appearing in Sec. 2) with either $u<v \leq r$ is maintained as a standard arc $(u, v, s) \in \mathscr{A}$, or with $u<r<v$ is transformed into a reflected arc $(u, L-v, r) \in \mathscr{A}$, or with $r \leq u<v$ is not considered anymore. Finally, we add the loss arcs $(d, e, l)$ (to connect all nodes $d, e$, where $e$ is the direct successor of $d \neq 0$ ) as well as the reflected arc $(L / 2, L / 2, r)$, see Fig. 1 for an example. Observe that the effect of raster points (as mentioned in Sect.2) is noticed almost only at the beginning of the graph, whereas it tends to be irrelevant for the second half of the vertices. Hence, the in- 


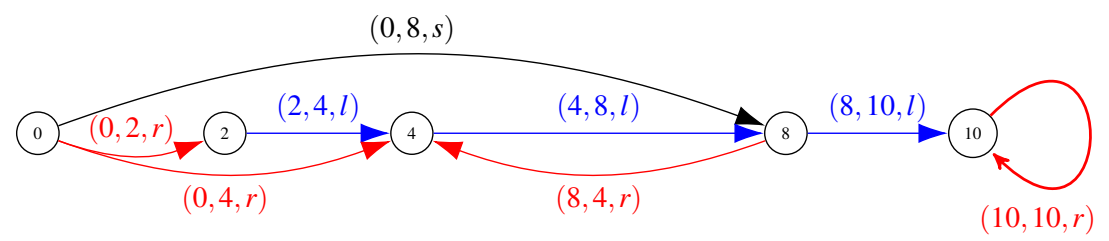

Fig. 1 The reflect graph for the instance $E=(3,(18,16,8), 20,(10,10,10))$. An optimal solution with $z^{R E}=15$ is given by $\xi_{0,2, r}=\xi_{0,4, r}=\xi_{0,8, s}=\xi_{2,4, l}=\xi_{8,10, l}=10, \xi_{4,8, l}=20$, and $\xi_{10,10, r}=-5$. The pattern $a^{1}=(0,2,0)^{\top}$ is used 5 times, and can be recomposed by two paths of type $(0,4, r) \rightarrow$ $(4,8, l) \rightarrow(8,10, l)$ that are connected by the reflected arc $(10,10, r)$. The pattern $a^{2}=(1,0,1)^{\top}$ is used 10 times and consists of the subpaths $(0,2, r) \rightarrow(2,4, l) \rightarrow(4,8, l)$ and $(0,8, s)$.

troduced restriction to the node set also significantly reduces the number of arcs compared to the original arcflow formulation.

Let us define $\mathscr{U}^{\star}:=\mathscr{U} \backslash\{0\}$ and $\mathscr{A}^{\star}:=\mathscr{A} \backslash\{(L / 2, L / 2, r)\}$, and let $\xi_{\text {deк }}$ denote the flow along the generic arc $(d, e, \kappa) \in \mathscr{A}$ (where $\kappa \in\{s, r, l\}$ is possible); then the reflect arcflow model is given by

\section{Reflect Arcflow Model of the SSP}

$$
\begin{aligned}
& z^{R E}=\max \sum_{(d, e, r) \in \mathscr{A}} \xi_{d e r} \\
& \text { s.t. } \sum_{(d, e, s) \in \mathscr{A}: e-d=l_{i}} \xi_{d e s}+\sum_{(d, e, r) \in \mathscr{A}: e=L-d-l_{i}} \xi_{d e r} \leq b_{i}, \\
& \sum_{(0, e, s) \in \mathscr{A}} \xi_{0 e s}+\sum_{(0, e, r) \in \mathscr{A}} \xi_{0 e r}=2 \sum_{(d, e, r) \in \mathscr{A}} \xi_{d e r}, \\
& \sum_{(d, e, s) \in \mathscr{A}} \xi_{d e s}+\sum_{(e, f, l) \in \mathscr{A}} \xi_{e f l}=\ldots \\
& \ldots \sum_{(d, e, r) \in \mathscr{A}} \xi_{d e r}+\sum_{(d, e, l) \in \mathscr{A}} \xi_{d e l}+\sum_{\kappa \in\{r, s\}} \sum_{(e, f, \kappa) \in \mathscr{A}} \xi_{e f \kappa}, \quad e \in \mathscr{U}^{\star}, \\
& \sum_{(d, e, l) \in \mathscr{A}} \xi_{d e l}+\sum_{(d, e, r) \in \mathscr{A}} \xi_{d e r} \geq \sum_{(e, f, l) \in \mathscr{A}} \xi_{e f l}, \quad e \in \mathscr{U}^{\star} \\
& \xi_{\text {deא }} \in \mathbb{Z}_{+}, \\
& (d, e, \kappa) \in \mathscr{A}^{\star}, \\
& \xi_{L / 2, L / 2, r} \in \mathbb{Z} \text {. }
\end{aligned}
$$

Since each unit of flow on a reflected arc $(d, e, r) \in \mathscr{A}$ refers to one single pattern, the objective function maximizes the number of constructed patterns. Moreover, conditions (4) require each item to be used at most $b_{i}$ times, whereas equation (5) basically says that two subpaths (starting at $v=0$ ) are needed per pattern (i.e., per unit of flow on a reflected arc). Constraints (6) model the flow conservation at interior vertices: each flow entering a node through a standard arc has to be continued by 
a flow leaving the node through a standard or a reflected arc (classical flow conservation), or by a flow entering the node through a reflected arc (connection between two subpaths), including the additional flow brought and removed by the loss arcs. Note that a loss arc may only follow another loss arc or a reflected arc, see (7). One particularity of this model is that we have to allow negative flows on the reflected $\operatorname{arc}(L / 2, L / 2, r)$ to enable two reflected paths to be merged together. This is required when large items have to be packed together in the optimal solution, see Fig. 1. Altogether, modeling and generating the reflect arcflow formulation for the SSP is more challenging than in the cutting scenario and requires (partly) independent techniques.

\section{Computational Results}

In order to evaluate the computational behavior of both models, we randomly generated 20 instances each for different pairs $(m, L)$ of input data (specified in Tab. 1) and collected the following averaged quantities: numbers of variables $n_{\text {var }}$ and constraints $n_{\text {con }}$, solution time $t$ (in sec.). The item lengths and availabilities were chosen from uniformly distributed integers $l_{i} \in[L / 10,3 / 4 \cdot L]$ and $b_{i} \in[1,100]$, respectively. All our experiments were executed on a Quad-Core AMD A10-5800K with $3.8 \mathrm{GHz}$ and 16 GB RAM, using Gurobi 7.5.2 as ILP solver.

Table 1 Averaged computational results for different choices of $(m, L)$

\begin{tabular}{l|l|r|r|r|r|r|r}
\multicolumn{2}{c}{} & \multicolumn{2}{c|}{$m=50$} & \multicolumn{2}{c|}{$m=100$} & \multicolumn{2}{c}{$m=200$} \\
\cline { 2 - 8 } \multicolumn{2}{c}{} & arcflow & reflect & arcflow & reflect & arcflow & reflect \\
\hline \multirow{3}{*}{$L=1000$} & $t$ & 0.43 & 0.08 & 2.11 & 0.32 & 10.07 & 2.20 \\
& $n_{\text {var }}$ & 8689.95 & 1473.30 & 23503.40 & 4729.10 & 62652.10 & 13253.75 \\
& $n_{\text {con }}$ & 711.75 & 634.20 & 871.80 & 954.90 & 1021.60 & 1163.80 \\
\hline \multirow{3}{*}{$L=2500$} & $t$ & 0.92 & 0.12 & 7.86 & 0.55 & 36.07 & 5.05 \\
& $n_{\text {var }}$ & 14265.60 & 1839.10 & 47986.25 & 6822.55 & 126413.55 & 20922.00 \\
& $n_{\text {con }}$ & 1486.00 & 1202.00 & 1866.90 & 1883.60 & 2135.90 & 2470.70 \\
\hline \multirow{3}{*}{$L=5000$} & $t$ & 1.86 & 0.15 & 20.41 & 1.02 & 101.62 & 9.13 \\
& $n_{\text {var }}$ & 20405.10 & 2080.25 & 74619.15 & 8377.60 & 210507.55 & 28205.00 \\
& $n_{\text {con }}$ & 2496.05 & 1621.55 & 3331.90 & 3094.00 & 3850.25 & 4290.10 \\
\hline
\end{tabular}

Based on the data from Tab. 1. it can clearly be seen that the original arcflow model is outperformed by the reflect formulation. Indeed, both models solved to proven optimality all attempted instances, but reflect has been much quicker. More precisely, for any of the 180 tested instances, the new approach led to significant savings in terms of variables (ranging from $77 \%$ to $92 \%$, with an average of $85 \%$ ) and solution times (ranging from 50\% to 97\%, with an average of $87 \%$ ). As regards the number 
of constraints, there is no clear relationship between the corresponding quantities. On the one hand, our new approach allows for using much fewer flow conservation constraints (in most cases only roughly $50 \%$ of the original number), whereas, on the other hand, an additional set of constraints (see (7p) has to be included.

\section{Conclusions}

In this paper we investigated an improved arcflow formulation for the skiving stock problem. The main idea of this approach is to only consider the first half of the vertex set, and to model a pattern as the sum of two subpaths that are connected by a reflected arc. As we have shown in the computational experiments, the new model possesses significantly fewer variables and much better solution times compared to the original formulation. Consequently, the reflect arcflow model may be seen as a powerful tool for solving (large) instances of the SSP in reasonably short time.

\section{References}

1. Assmann, S.F., Johnson, D.S., Kleitman, D.J., Leung, J.Y.-T.: On a dual version of the onedimensional Bin Packing Problem. J. Algorithms 5, 502-525 (1984)

2. Chen, Y., Song, X., Ouelhadj, D., Cui, Y.: A heuristic for the skiving and cutting stock problem in paper and plastic film industries. Int. Trans. Oper. Res. 26(1), 157-179 (2019)

3. Côté, J.-F., Iori, M.: The Meet-in-the-Middle Principle for Cutting and Packing Problems. INFORMS J. Comput. 30(4), 646-661 (2018)

4. Christofides, N., Whitlock, C.: An Algorithm for Two-Dimensional Cutting Problems. Oper. Res. 25(1), 30-44 (1977)

5. Delorme, M., Iori, M.: Enhanced Pseudo-Polynomial Formulations for Bin Packing and Cutting Stock Problems. Technical Report OR-17-6, DEI "Guglielmo Marconi”, Università di Bologna (2017)

6. Delorme, M., Iori, M., Martello, S.: Bin Packing and Cutting Stock Problems: Mathematical Models and Exact Algorithms. Eur. J. of Oper. Res. 255, 1-20 (2016)

7. Johnson, M.P., Rennick, C., Zak, E.J.: Skiving addition to the cutting stock problem in the paper industry. SIAM Rev. 39(3), 472-483 (1997)

8. Martinovic, J., Jorswieck, E., Scheithauer, G., Fischer, A.: Integer Linear Programming Formulations for Cognitive Radio Resource Allocation. IEEE Wireless Commun. Lett. 6(4), 494497 (2017)

9. Martinovic, J., Scheithauer, G.: Integer linear programming models for the skiving stock problem. Eur. J. of Oper. Res. 251(2), 356-368 (2016)

10. Martinovic, J., Scheithauer, G., de Carvalho, V.: A Comparative Study of the Arcflow Model and the One-Cut Model for one-dimensional Cutting Stock Problems. Eur. J. of Oper. Res. 266(2), 458-471 (2018)

11. Nemhauser, G., Wolsey, L.: Integer and Combinatorial Optimization. Wiley, New York (1988)

12. Scheithauer, G.: Introduction to Cutting and Packing Optimization - Problems, Modeling Approaches, Solution Methods. Springer, Chur (2018)

13. Valério de Carvalho, J.M.: LP models for bin packing and cutting stock problems. Eur. J. of Oper. Res. 141(2), 253-273 (2002)

14. Zak, E.J.: The skiving stock problem as a counterpart of the cutting stock problem. Int. Trans. Oper. Res. 10, 637-650 (2003) 